\title{
Nutracêuticos e seus benefícios para a saúde do utente: revisão integrativa da
}

\section{literatura}

\author{
Nutraceutics and their benefits for user health: integrative literature review \\ La nutracéutica y sus beneficios para la salud del usuario: revisión integrativa de la literatura
}

Recebido: 08/09/2021 | Revisado: 16/09/2021 | Aceito: 27/09/2021 | Publicado: 27/09/2021

\author{
Brendo Borges Magalhães de Alencar \\ ORCID: https://orcid.org/0000-0002-2821-302X \\ Faculdade Integrada Carajás, Brasil \\ E-mail: brendobma@ @otmail.com \\ Yolanda de Jesus Morais \\ ORCID: https://orcid.org/0000-0001-7105-8267 \\ Faculdade Integrada Carajás, Brasil \\ E-mail: yolandamorais123@gmail.com
}

\begin{abstract}
Resumo
Introdução: O índice elevado da expectativa de vida tem gerado um grande interesse em todo o mundo sobre a alimentação saudável. Nesse sentido, vem acontecendo um importante acréscimo do cultivo de alimentos específicos, com o intuito de promover a equilíbrio e à manutenção da saúde. Esse artigo tem como objetivo identificar produções bibliográficas sobre os potenciais benefícios dos nutracêuticos para a saúde do utente, descrevendo o conhecimento produzido acerca desta temática. Metodologia: Trata-se de uma revisão integrativa da literatura baseada na pesquisa bibliográfica. As estratégias de busca permitiram a identificação de 366 artigos nas bases de dados selecionadas. Ao longo das análises dos títulos e resumo, aplicando os critérios de inclusão, 360 artigos foram excluídos. Ao total foram selecionados 06 artigos para análise do texto integral que participaram da revisão sobre o assunto. Utilizando critérios de inclusão e exclusão de artigos, após a leitura e aplicação dos critérios selecionados, 06 artigos foram selecionados por atenderem todos os requisitos. Resultados e discussão: Os resultados apontam que os nutracêuticos possuem benefícios importantes para a saúde do utente e que podem ser utilizados como uma terapia complementar, com a introdução de hábitos saudáveis para a melhoria da qualidade de vida. Considerações finais: Mais pesquisas devem ser realizadas com o intuito de traçar os reais benefícios dos nutracêuticos.
\end{abstract}

Palavras-chave: Nutracêuticos; Benefícios dos nutracêuticos; Suplemento alimentar.

\begin{abstract}
Introduction: The increase in life expectancy has aroused greater interest in the topic of healthy eating. With this, there is an important increase in the production of specific foods, aimed at maintaining health. This article aims to identify bibliographic productions on the potential benefits of nutraceuticals for the user's health, describing the knowledge produced on this subject. Methodology: This is an integrative literature review based on bibliographic research. The search strategies allowed the identification of 366 articles in the selected databases. Throughout the analysis of titles and abstract, applying the inclusion criteria, 360 articles were excluded. In total, 06 articles were selected for analysis of the full text that participated in the review on the subject. Using criteria for inclusion and exclusion of articles, after reading and applying the selected criteria, 06 articles were selected for meeting all requirements. Results and discussion: The results show that nutraceuticals have important benefits for the user's health and that they can be used as a complementary therapy, with the introduction of healthy habits to improve the quality of life. Final considerations: More research should be carried out in order to trace the real benefits of nutraceuticals.
\end{abstract}

Keywords: Nutraceuticals; Benefits of nutraceuticals; Food supplement.

\section{Resumen}

Introducción: El aumento de la esperanza de vida ha despertado un mayor interés en el tema de la alimentación saludable. Con esto, hay un aumento importante en la producción de alimentos específicos, orientados a mantener la salud. Este artículo tiene como objetivo identificar producciones bibliográficas sobre los potenciales beneficios de los nutracéuticos para la salud del usuario, describiendo el conocimiento producido sobre este tema. Metodología: Se trata de una revisión integradora de la literatura basada en la investigación bibliográfica. Las estrategias de búsqueda permitieron identificar 366 artículos en las bases de datos seleccionadas. A lo largo del análisis de títulos y resúmenes, aplicando los criterios de inclusión, se excluyeron 360 artículos. En total, se seleccionaron 06 artículos para el análisis del texto completo que participaron en la revisión sobre el tema. Utilizando criterios de inclusión y exclusión de artículos, después de leer y aplicar los criterios seleccionados, se seleccionaron 06 artículos que cumplían con todos los requisitos. Resultados y discusión: Los resultados muestran que los nutracéuticos tienen importantes beneficios para la salud del usuario y que pueden utilizarse como terapia complementaria, con la introducción de hábitos 
saludables para mejorar la calidad de vida. Consideraciones finales: Se deben realizar más investigaciones para rastrear los beneficios reales de los nutracéuticos.

Palabras clave: Nutracéuticos; Beneficios de los nutracéuticos; Suplemento alimenticio.

\section{Introdução}

Os nutracêuticos podem ser definidos como alimentos, ou parte destes, os quais apresentam benefícios à saúde. Um nutracêutico também é considerado um farmaconutriente, e contém em sua composição nutriente que pode potencializar, combater, ou modificar funções fisiológicas ou metabólicas (Souza, 2008; Fernandes, 2011; Barcarol et al., 2012).

A ingestão de micronutrientes pode prevenir manifestações patológicas e estados de carência nutricional, as quais podem ter um impacto significativo em pacientes que apresentam patologias. Nesse contexto, os nutracêuticos tem se tornado poderoso instrumento como alternativa na manutenção da saúde, contra agravos nutricionais e melhora na qualidade de vida dos usuários (Lira et al., 2009; Fernandes, 2011; Vieira et al., 2013).

Diante desse cenário, nesse estudo deparamo-nos com uma pergunta a ser pesquisada: quais os potenciais benefícios oriundos da utilização dos nutracêuticos para a saúde do utente?

Este estudo busca dados dentro do contexto da utilização dos nutracêuticos, partindo-se da hipótese de que o seu uso gera benefícios para a saúde do utente.

O estudo justifica-se pela expansão em que se encontra o mercado mundial de nutracêutico, com vendas em largo crescimento, especialmente no varejo farmacêutico. De acordo com o Instituto de Ciência e Tecnologia da Qualidade Industrial (ICTQ), desde 2015, indústrias voltadas ao segmento nutracêutico, obtiveram faturamentos expressivos. Dessa forma, torna-se imprescindível reconhecer os potenciais benefícios dos nutracêuticos.

Objetiva-se com esse artigo identificar as produções bibliográficas sobre esse tema que tanto tem impulsionado o mercado mundial, na busca de melhorar a qualidade de vida da população, destacando seus potenciais benefícios a saúde.

\section{Nutracêuticos}

A evolução da ciência da nutrição permitiu que, na atualidade, muito mais se saiba sobre a ação de cada nutriente no organismo e, além disso, vem demonstrando que algumas substâncias normalmente presentes nos alimentos, denominadas compostos bioativos $(\mathrm{CB})$, mas ainda não consideradas como nutrientes, podem ser importantes para redução do risco de doenças e também para o tratamento (Cazzolino, 2012).

Zeisel (1999), definiu nutracêuticos como suplementos alimentares que contêm a forma concentrada de um CB de alimento, exibido separadamente da matriz alimentar e empregado com a finalidade de melhorar a saúde, em doses que excedem aquelas que poderiam ser alcançadas de alimentos.

Dessa forma, Hipócrates, o reconhecido pai da medicina moderna, definiu a relação entre o uso de alimentos apropriados para a saúde e seus benefícios terapêuticos através da frase: "Deixe o alimento ser o medicamento, e o medicamento ser o alimento" (Bagchi, 2006; Wildman, 2000).

Os nutracêuticos podem ser dispostos em distintos grupos, conforme o interesse envolvido - fonte alimentar, propriedade funcional e natureza química (Suleria et al., 2015; Stringueta, 2007).

Mediante a fonte alimentar os nutracêuticos são classificados em grupo vegetal (ex.: licopeno), animal (ex.: DHAÁcido Docosa-hexanóico), bactérias e leveduras (ex.: Bifidobacterium bifidum; Saccharomyces boulardii). Esta classificação pode ser um instrumento valioso em termos de planejamento alimentar (Kokate; Purohit; Gokhale, 2009; Stringueta, 2007).

Suas propriedades funcionais se agrupam com base em suas propriedades fisiológicas provadas ou pretendidas desconsiderando a fonte alimentar. Sendo classificadas como antioxidante; antibacteriano, hipotensivo, anti-inflamatório, 
anticarcinogênico, protetor ósseo, hipocolesterolêmico, imunomodulador dentre outras (Cardoso; Oliveira, 2008; Stringueta, 2007).

Diversos CBs, vêm sendo comercializados na forma de produtos farmacêuticos, como cápsulas, soluções, géis, pós e granulados. Esse grande número de produtos não pode ser genuinamente classificado como alimentos, por essa razão usa-se o um termo híbrido de nutrientes e farmacêuticos (Espín et al., 2007).

\section{Legislação dos nutracêuticos}

O termo nutracêutico tem sido muito debatido em todo o mundo, entretanto, ainda não há uma consonância mundial sobre sua definição, sendo, portanto, utilizada diversas terminologias para sua classificação (Rajasekaran et al; 2015).

Apesar de não estarem regulamentados com esta denominação os nutracêuticos encontram-se vinculados as legislações de alimentos funcionais (Pimentel, Francki, Gollücke, 2005).

Conforme a Resolução no. 18 (Estabelece as Diretrizes Básicas para Análise e Comprovação de Propriedades Funcionais e ou de Saúde Alegadas em Rotulagem de Alimentos) (ANVISA, 1999) e a Resolução no 19, de 30 de abril de 1999 (Estabelece as alegações de procedimentos para registro de alimento com alegação de propriedades funcionais e/ou de saúde) (ANVISA, 1999).

Além disso, também podem ser enquadrados através das seguintes portarias: Portaria SVS/MS nº 32/98 (Regulamenta Suplementos Vitamínicos e, ou Minerais) e Portaria SVS/MS n 31/98 (Regulamenta Alimentos Adicionados de Nutrientes Essenciais) (Carvalho, 2004; Stringheta et al., 2007).

A ANVISA através das resoluções certifica a qualidade, segurança e a eficácia dos medicamentos produzidos, tanto nas indústrias como nas farmácias magistrais (Pineze et al., 2003). A resolução RDC nº 214, de 12 de dezembro de 2006 da ANVISA, por exemplo, foi criada com a finalidade de fixar os requisitos mínimos exigidos para a manipulação, fracionamento, conservação, transporte e dispensação de produtos de interesse da saúde (Brasil, 2006).

Recentemente, o Conselho Federal de Farmácia através da resolução № 661, de 25 de outubro de 2018, instituiu o cuidado farmacêutico pertinente a suplementos alimentares e demais categorias de alimentos na farmácia comunitária, consultório farmacêutico e estabelecimentos comerciais de alimentos. A resolução enfatiza a importância do profissional farmacêutico na orientação e prescrição dos nutracêuticos.

Os estudos farmacodinâmicos revelam o comportamento do nutracêutico no organismo, desta forma, analisam o sítio de ação da substância presente no nutracêutico (Lira, 2009). Favorecem, além disso, uma comparação entre as respostas humana e animal à dose administrada fornecendo informações sobre uma provável toxicidade do produto (Brasil, 1996).

O registro de um nutracêutico acontecerá após constatação das suas propriedades funcionais a saúde com base no consumo previsto ou recomendado pelo fabricante, finalidade e condições de uso com valor nutricional, comprovado e evidência(s) científica(s): composição química/caracterização molecular, na formulação do produto; teses bioquímicos; teses nutricionais e ou fisiológicos e ou toxicológicos em animais de experimentação; estudos epidemiológicos; teses clínicos; evidências abrangentes da literatura científica, organismos internacionais de saúde e legislação internacionalmente reconhecidas sob propriedades e características do produto e comprovação de uso tradicional, observado na população, sem associação de danos à saúde (Brasil, 1999; Brasil 1999; Pimentel, et al.,2005).

\section{Nutracêuticos e seus benefícios}

Souza (2017), destaca que mediante os paradigmas contemporâneos pertinentes aos cuidados com a saúde, onde o ser humano encontra-se mais voltado à promoção da saúde, do bem-estar e da diminuição do risco de algumas doenças, as ciências 
alimentares passaram a desenvolver produtos que corroboram com este novo paradigma, proporcionando distintos benefícios aos utentes por meio das opções nutricionais adequadas produzidas pelos nutracêuticos.

\section{Propriedade antioxidante}

De modo geral antioxidante é "qualquer substância que, presente em baixas concentrações quando comparada a do substrato oxidável, atrasa ou inibe a oxidação deste substrato de maneira eficaz" (Sies \& Stahl, 1995).

A oxidação pode ocorrer devido à ação de radicais livres presentes no organismo. Pode provocar lesões nas células, as quais podem ser prevenidas ou reduzidas por meio da atividade de agentes antioxidantes. Os antioxidantes podem agir diretamente na neutralização da ação dos radicais livres. Dentre os antioxidantes estão: a vitamina C (ácido ascórbico), a glutationa, a vitamina E (tocoferóis), os carotenoides, zinco, selênio, cobre, vitamina A (retinol), flavonóides, curcumina, beta caroteno e licopeno (Moraes \& Colla, 2006).

Entre estes destacam as vitaminas $\mathrm{C}$ e E que são consideradas excelentes antioxidantes, capazes de sequestrar os radicais livres com grande eficiência (Nedel, 2005; Bianchi et al., 1999).

A vitamina $\mathrm{E}$ em sua forma ativa $\alpha$-tocoferol, é encontrado em lipoproteínas e membranas, agindo no bloqueio da reação em cadeia da peroxidação lipídica, através do sequestro do radical peroxila (Sousa et al., 2007).

A vitamina C (ácido ascórbico) é agente de reações de hidroxilação da prolina, indispensável para a formação do colágeno nos tecidos (Gonçalves et al., 2013). Este ácido participa ainda da biossíntese de corticosteróides e de carnitina, além de contribuir na absorção de ferro (Rotta, 2003).

\section{Propriedade imunomoduladora}

São agentes que atuam modulando e/ou ativando os componentes celulares e seus mediadores químicos, como consequência, aumentam a efetividade do sistema imune contra diferentes antígenos, evitando o aparecimento de possíveis patologias no organismo. Dentre as substâncias imunomoduladoras, encontram-se os compostos químicos presentes em frutas, cereais, hortaliças e chás, como os carotenoides e compostos fenólicos (catequinas, flavonóides) (Souza, 2008).

A arginina é um aminoácido semiessencial, que se torna essencial quando ocorre alguma alteração no metabolismo. Seu efeito farmacológico apresenta ser exclusivamente sobre o sistema imunológico, através de proliferação do linfócito T (Zhou, 2007). A glutamina é aminoácido condicionalmente essencial, exercendo efeito benéfico na função imune através da produção de linfócitos T e B e imunoglobulina A (IgA) (Grimble, 2005; Stephen, 2009). A glutamina também é fonte energética importante para os macrófagos, linfócitos e todas as células do sistema imunológico (Hasenboehler, 2006).

\section{Controle da obesidade}

Atualmente, a obesidade é um problema de saúde pública, visto que reduz a qualidade de vida e aumenta consideravelmente o gasto público com a saúde. Os nutracêuticos têm se tornado uma opção eficaz no tratamento da obesidade, principalmente por possuírem componentes químicos como flavonoides, alcaloides, terpenos, dentre outros, que favorecem o emagrecimento (Bernardi et al., 2011; Teixeira et al., 2014).

Nesse contexto é válido destacar a importância do óleo de coco, que vem sendo largamente utilizado com a promessa de possuir excelente ação antibacteriana, antiviral e antifúngica, o que ajudaria no combate a vários microrganismos patogênicos. Esse possível efeito terapêutico do óleo de coco estaria relacionado à presença de um composto, o ácido láurico, que apresenta efeito termogênico. É composto também por fitoesteróis que atuam promovendo a diminuição da absorção de colesterol no intestino delgado (Caleiro, 2012; Dauber, 2015). 
O óleo de cártamo também é citado no controle da obesidade, pois desempenha potente ação antioxidante, promove a saciedade, inibe o apetite, e busca essencialmente a prevenção e o tratamento de hiperlipidemias. Além disso, o óleo de cártamo estimula o sistema imunológico e reduz a massa corporal, o que promove diminuição da obesidade (Ambrosi et al., 2008; Campanella et al., 2014; Schulze et al., 2014).

A L - Canitina, favorece a diminuição da lipoproteína de baixa densidade (LDL), bem como a diminuição dos níveis de açúcar no sangue e da hipertensão arterial sistêmica (HAS). Já o ácido linoléico conjugado (CLA), favorece a diminuição da massa gorda corporal e a diminuição de peso a longo prazo, é encontrado principalmente na gordura e no leite de ruminantes, indicando que a carne bovina e laticínios são sua principal fonte (Alegre, 2015; Nogueira, 2015).

A fibra conhecida como glucomanano (Amorphophallus konjac) apresenta viscosidade excepcionalmente elevada. Esta forma um gel que rapidamente aumenta o teor em umidade do bolo alimentar durante a digestão, podendo aumentar a saciedade, diminuir o apetite e fazer com que o indivíduo coma menos a cada refeição, reduzindo assim o peso corporal. Outro nutracêutico largamente utilizado no controle da obesidade e na perda de peso corporal é a quitosana, derivada da quitina, que também apresenta ação antiobesidade (Silva, 2013; Alegre, 2015).

\section{Propriedade anticancerígena}

O câncer tem se tornado, cada vez mais, uma das principais causas de morte em todo o mundo diante disto, os nutracêuticos podem ser utilizados na prevenção, do câncer colorretal, visto que estes podem prevenir a formação de pólipos ou adenomas no intestino que é o terceiro tipo mais comum no mundo todo, e suas causas estão diretamente ligadas ao estilo de vida e dieta. Dentre os principais nutracêuticos com propriedade anticancerígena está a Spirulina Spp., que apresenta perfil nutricional e possui $\beta$-caroteno, precursor da vitamina A, em sua composição (Ambrosi et al., 2008; Markle et al., 2010).

Dente os alimentos contendo agentes para a quimioprevenção estão os alimentos que apresentam em sua composição propriedade antioxidante (como o ácido ascórbico, fitoestrógenos da soja e carotenóides), os agentes indutores de apoptose (morte celular programada), os inibidores de enzima do Citocromo P450 que é responsável pelo metabolismo de drogas, e que neste processo pode, de forma involuntária, favorecer a formação de radicais livres carcinogênicos, os agentes inibidores enzimáticos e inibidores da angiogênese entre outros. Um importante agente anticarcinogênico é o CLA, o qual apresenta efeitos anticarcinogênicos e antiaterogênicos (Ferrari \& Torres, 2002; Alegre, 2015).

\section{A importância do profissional farmacêutico na orientação quanto ao uso correto dos nutracêuticos}

O Farmacêutico tem um papel fundamental na promoção da saúde pública e no uso racional do medicamento. Tendo a responsabilidade de orientar o utente quanto ao uso correto do medicamento e também apresentar ao utente as características pertinentes a cada medicamento utilizado por ele (Lopes, 2013).

O elo criado entre o farmacêutico e o utente, facilita a intervenção do farmacêutico em relação a utilização dos nutracêuticos pelo utente, beneficiando o uso racional dos nutracêuticos que na maioria das vezes são utilizados, por iniciativa própria dos utentes, daí sobrevém a importância da intervenção farmacêutica.

O Farmacêutico deve esclarecer todas as dúvidas colocadas pelos utentes, enfatizando a papel dos nutracêuticos num regime alimentar equilibrado, como agentes promotores de saúde e não como substitutos da alimentação (Ferraz \& Pinto, 2009).

Cabe ao Farmacêutico aconselhar sobre as opções disponíveis, informar sobre as condições de utilização e de administração, bem como dispensar ou não o nutracêutico, se por ventura constatar que a sua administração não é segura ou necessária. 
Deve-se alertar o utente que os nutracêuticos são CB que alteram as funções próprias do organismo e que podem alterar a farmacocinética e a farmacodinâmica dos medicamentos que o utente possa estar fazendo o uso concomitantemente (Campos, 2012).

\section{Metodologia}

O estudo trata-se de uma revisão integrativa da literatura (Cunha, Cunha \& Alves, 2014), que se baseia na pesquisa bibliográfica que é um método que proporciona a síntese de conhecimentos e a incorporação da aplicabilidade de resultados e estudos significativos na prática.

As etapas seguidas para a elaboração da revisão integrativa foram as seguintes: inicialmente construiu-se a hipótese e o objetivo do estudo, em seguida selecionou-se a amostra por meio dos critérios de inclusão bem como: artigos científicos que não tenha como metodologia de estudo revisão da literatura sem limitação de idioma, que estejam disponíveis eletronicamente na íntegra.

A definição da amostra e a coleta de dados estruturada ocorreu no período de julho e agosto de 2021, com busca nas bases de dados virtual Literatura Latino- Americana e do Caribe em Ciências da Saúde (LILACS), Scientific Eletronic Library Online (SciELO), BVS (Biblioteca Virtual em Saúde). O levantamento dos artigos foi realizado utilizando os seguintes DeCs (Descritores em Ciências da Saúde): nutracêuticos, benefícios dos nutracêuticos e suplemento alimentar.

Ao término da busca dos artigos, considerando os critérios de inclusão, os artigos foram armazenados em computador, e posteriormente realizou-se uma pré-seleção mediante a leitura dos resumos. Nessa etapa verificou-se a semelhança entre os conteúdos, títulos, resumos e se os mesmos atendem ao objetivo geral do estudo.

$\mathrm{Na}$ etapa de seleção os artigos foram lidos na íntegra com atenção especial para os resultados e conclusão dos estudos. Os estudos que por sua vez não exibiram qualquer relação com os nutracêuticos foram excluídas.

A etapa de análise dos resultados foi realizada por meio da disposição e apresentação em forma de tabelas do Microsoft Office Word 2010, essa fase da pesquisa teve como finalidade sumarizar os resultados relacionados e em seguida discuti-los.

As informações retiradas dos artigos foram: autores, ano de publicação, cidade onde foi realizado o estudo, benefícios dos nutracêuticos, desenho do estudo, metodologia e resultados. Por último, realizou-se a análise dos textos, a leitura interpretativa e a redação.

Mediante os critérios estabelecidos para as buscas foram identificados 366 artigos nas bases de dados selecionadas. De acordo com as análises dos títulos e resumos um total de 336 artigos foram excluídos por diversos motivos dentre eles: assuntos não condizentes com o abordado, ausência da apresentação de resumos ou artigos na íntegra. Ao final foram selecionados 6 artigos para apreciação do texto integral que participaram da revisão sobre o assunto.

A Figura 1 elucida a estratégia de busca e a metodologia de seleção dos artigos. 


\section{Figura 1.}

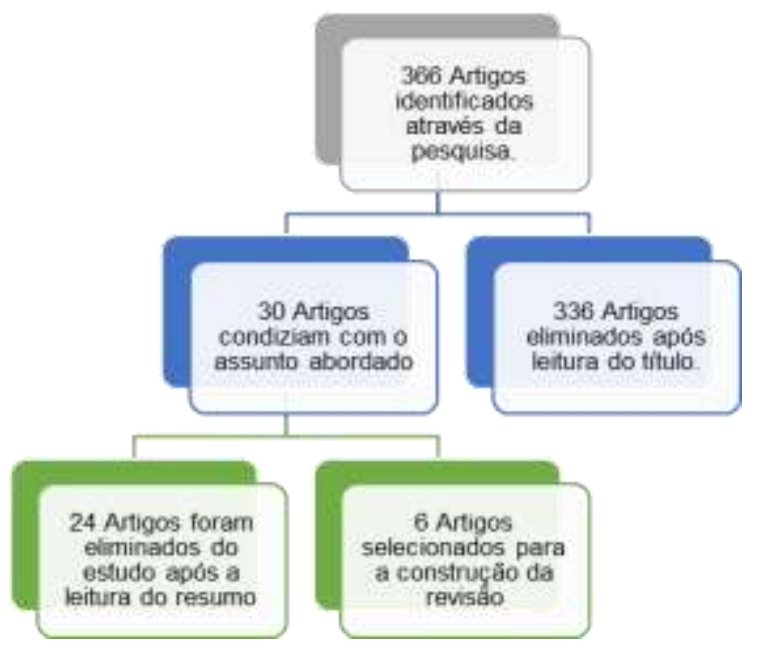

Fonte: Nutracêuticos e seus benefícios para a saúde do utente: Um estudo de revisão.

\section{Resultados e Discussão}

A Tabela 1 apresenta detalhes dos 6 artigos selecionados. Todos os estudos foram publicados entre 2015 e 2019, onde foram analisados os potenciais benefícios dos nutracêuticos. Mediante as buscas pode-se notar que o número de publicações ainda é pouco expressivo, principalmente a nível nacional, uma vez que dos 6 artigos analisados apenas 3 foram conduzidos no Brasil, a ausência de pesquisas na área estudada relatada por diversos autores, dificulta traçar o real benefício dos nutracênticos mesmo esses produtos estando em grande ascensão no mercado farmacêutico. 
Research, Society and Development, v. 10, n. 12, e484101220396, 2021

(CC BY 4.0) | ISSN 2525-3409 | DOI: http://dx.doi.org/10.33448/rsd-v10i12.20396

Tabela 1. Detalhes dos 06 artigos selecionados.

\begin{tabular}{|c|c|c|}
\hline ARTIGO & OBJETIVO & DESENHO DO ESTUDO \\
\hline Neves \& Saunders (2015) & $\begin{array}{l}\text { Avaliar o impacto da suplementação com vitamina A (VA) em gestantes e puérperas adultas no Brasil sobre o } \\
\text { teor de VA e imunoglobulina A secretora (IgAS) do colostro e leite materno, nas condições } \\
\text { de saúde infantil e no status de VA do binômio mãe-filho. }\end{array}$ & Retrospectivo \\
\hline Rumbold \& Ota (2016) & $\begin{array}{l}\text { Avaliar os efeitos da suplementação de vitamina E, isoladamente ou em combinação com outros suplementos } \\
\text { separados, na gravidez. }\end{array}$ & Retrospectivo \\
\hline Santos \& Gonçalves (2016) & $\begin{array}{l}\text { Avaliar os potenciais benefícios da suplementação de AGPI } \omega- \\
3 \text { em pacientes com doenças cardiovasculares }\end{array}$ & Retrospectivo \\
\hline Kumar \& Yadav (2017). & Avaliar os potenciais benefícios da suplementação de vitamina D na função vascular na DRC & Randomizado \\
\hline Schalka \& Bombarda (2018) & $\begin{array}{l}\text { Avaliar a eficácia de nutracêutico contendo, entre outros elementos, vitaminas A, C, E, complexo B, ácido fólico, } \\
\text { ferro, niacina, biotina, zinco e pantetonato de cálcio, no tratamento do eflúvio telógeno associado à síndrome das } \\
\text { unhas fracas. }\end{array}$ & Unicêntrico \\
\hline Han \& Jo (2019) & $\begin{array}{l}\text { Avaliar a eficácia do uso do resveratrol supressor de tumores microambiente tumoral modulador e maligno nos } \\
\text { comportamentos do } \\
\text { Câncer }\end{array}$ & Retrospectivo \\
\hline
\end{tabular}

Fonte: Nutracêuticos e seus benefícios para a saúde do utente: Um estudo de revisão. 
No estudo de Neves e Saunders (2015), de natureza retrospectiva, observacional e descritivo, conduzido no Brasil, com finalidade de avaliar o impacto da suplementação com vitamina A em gestantes e puérperas adultas no Brasil. Os autores puderam identificar através dos resultados obtidos, que a suplementação de vitamina A no puérpero elevou o teor de retinol no leite materno, aumentando assim a oferta de vitamina A para a criança e a concentração de imunoglobulina IgA, no colostro, Lima et al., (2012), destaca que esse dado representa uma vantagem extremamente importante principalmente para os lactentes, uma vez que as imunoglobulinas apresentam um papel um papel importante na proteção contra infecções que podem acometer o RN, destacando assim o papel da vitamina A no sistema imunológico.

Rumbold e Ota (2016), em seu estudo conduzido no Brasil, avaliaram os potenciais benefícios da suplementação de AGPI $\omega$-3 em pacientes com doenças cardiovasculares por meio de um estudo retrospectivo e descritivo, onde os autores puderam observar uma diversidade de benefícios a saúde do utente bem como, a melhoria dos parâmetros cardíacos na coagulação do sangue, concentração de triglicerídeos, concentração de leptina e deponectina, melhora de sintomas depressivos e redução da concentração plasmática de citocinas inflamatórias, os autores descrevem ainda que as dificuldades de se obter resultados demonstrados os benefícios dos AGPI se deve a melhoria do tratamento medicamentoso nas doenças cardiovasculares, deste modo a suplementação é indicada para pacientes com doenças cardiovasculares, como uma terapia adjuvante ao tratamento e as mudanças de estilo de vida.

Rumbold e Ota (2016), conduziram um estudo na Austrália, onde foi avaliado os efeitos da suplementação de vitamina E, isoladamente ou em combinação com outros suplementos separados na gravidez. Por meio de um estudo retrospectivo e descritivo os autores identificaram através dos resultados obtidos, que a suplementação rotineira com vitamina E em gestantes está associada ao risco aumentado, de dor abdominal auto referida, não sendo constatado resultados positivos na prevenção de morte neonatal ou parto prematuro, os autores ainda destacam que não haviam evidências suficientes para provar o benefício da suplementação da vitamina E na prevenção do deslocamento de placenta.

Kumar e Yadav (2017), investigaram o efeito da suplementação de vitamina D na função vascular em 120 pacientes de ambos os sexos entre 18 e 70 anos, com DRC (Doença Renal Crônica) não diabética, nesse estudo os autores forneceram fortes evidências de que a suplementação de vitamina D é segura e pode ser utilizada redução do risco CV (cardiovascular) em pacientes com DRC e deficiência de vitamina D.

Os autores destacam ainda que poucas são as pesquisas que abordam a suplementação com vitamina $\mathrm{D}$ em pacientes com DRC. Em virtude desse fato, entende-se que ainda é reduzido o número de pesquisadores que se envolvem com essa temática. Porém, os resultados positivos no uso da suplementação de vitamina D contribuem para um melhor manejo terapêutico desses pacientes com DRC.

Schalka e Bombarda (2018), no estudo conduzido no Brasil através de um estudo unicêntrico aberto e prospectivo com a participação de 62 voluntárias, puderam avaliaram a eficácia do nutracêutico associado a síndrome das unhas fracas. Observando ao final do estudo uma melhoria significativa nos parâmetros clínicos relacionados aos cabelos e as unhas, mediante o uso do nutracêutico.

Finner (2016), destaca que o uso de suplementação vitamínica e mineral nas patologias capilares é empregada de longa data com distintos registros na literatura. Especial atenção é dada aos CB presentes nos micronutrientes, incluindo minerais, vitaminas e aminoácidos. Estando os fios capilares compostos em 98\% de proteínas, presume-se que uma dieta rica em proteínas seja essencial para sua vitalidade. De fato, em estados nutricionais em que há carência na ingesta de proteínas, os fios capilares são visivelmente afetados.

Han e Jo (2019), puderam avaliar a eficácia do uso do nutracêutico resveratrol supressor de tumores microambiente tumoral modulador e maligno no comportamento do câncer. De acordo com o estudo revisado, o uso do nutracêutico resveratrol tem demonstrado a supressão tumoral, regulando o microambiente de tumores, suprimindo assim a progressão do 
câncer. Através de um estudo retrospectivo os autores identificaram que o uso do resveratrol tem demonstrado uma positiva supressão tumoral. Tornando-se cada vez mais evidente que o resveratrol também pode regular a microambiente tumoral, suprimindo a progressão do câncer. Mendoza-Rodriguez et al., (2017), destacam ainda que os aspectos do microambiente do tumor, é formado de diversas condições celulares e não celulares, por essa razão torna-se necessário e entender melhor a eficácia do resveratrol e de outros compostos naturais na progressão do câncer.

\section{Considerações Finais}

Através da presente revisão foi possível constatar que os nutracêuticos podem ser utilizados como um complemento da terapia medicamentosa, pois auxiliam na melhoria da qualidade de vida, uma vez que tais produtos proporcionam diversos benefícios à saúde. Isso desperta um marcante interesse mundial quanto ao aumento mercadológico, em termos de lucratividade.

Entretanto, considerando técnicas e instrumentos propostos nos estudos incluídos, não se pode constar uma homogeneidade por se tratar de produtos que ainda não tem uma legislação específica, diante disso, esses produtos requerem uma legislação mundial que viabilize adequadamente sua definição, bem como, garantir a sua segurança, qualidade e eficácia.

Contudo, ressalta-se que demais estudos necessitam ser realizados a fim de estabelecer os reais benefícios da utilização dos nutracêuticos.

\section{Contribuições dos autores}

BBMA concebeu a estratégia de pesquisa em conjunto com YJM. BBMA fez as buscas bibliográficas nas bases de dados, a seleção dos artigos e a extração de dados sob a supervisão de YJM. Os autores leram e aprovaram a versão final do manuscrito. O conteúdo da revisão é de exclusiva responsabilidade individuais dos autores.

\section{Declaração de conflito de interesses}

Os autores declaram que a pesquisa foi conduzida na ausência de quaisquer relações comerciais ou financeiras que possam ser interpretados como um potencial conflito de interesse.

\section{Referencias}

Alegre, A. D. S. (2015). Suplementos alimentares para gestão de peso corporal: riscos e benefícios. https://repositorio.ipv.pt/handle/10400.19/3192.

Ambrosi, M. A., Reinehr, C. O., Bertolin, T. E., Costa, J. A. V., \& Colla, L. M. (2008). Propriedades de saúde de Spirulina spp. Revista de Ciências Farmacêuticas Básica e Aplicada, 29(2). http://rcfba.fcfar.unesp.br/index.php/ojs/article/view/477.

Bagchi, D. (Ed.). (2019). Nutraceutical and functional food regulations in the United States and around the world. Academic press. https://books.google.com.br/books?hl=pt-BR\&lr=\&id=q3ScDwAAQBAJ\&oi=fnd\&pg=PP1\&dq=Debasis.+Nutraceuticals+and+func tional+foods+ regulatio ns+in+the+United+States+and+around+the+world.+Toxicology\&ots=Nx8148951P\&sig=eqjz6YL_yQ616mvtFZHzFER15I0\#v=onepage\&q=Debasis.\%20Nut raceuticals $\% 20$ and $\% 20$ functional $\% 20$ foods $\% 20$ regulations $\% 20$ in $\% 20$ the $\% 20$ United $\% 20$ States $\% 20$ and $\% 20$ around $\% 20$ the $\% 20$ world. $\% 20$ Toxicology \&f $=$ false

Barcarol, L. N., Santos, M. R., Rubin, R. H., Lima, C. P., Bonatto, J. G. B., Rech, L. S., \& Marisco, P. D. C. (2012). Alimentos funcionais e nutracêuticos. XVI Seminário Interinstitucional de Ensino, Pesquisa e Extensão. Unicruz, $1 . \quad$ https://home.unicruz.edu.br/seminario/anais/anais2012 /ccs/alimentos\%20funcionais\%20e\%20nutraceuticos.pdf.

Bernardi, M. M. (2011). A eficácia e Segurança do Insumo Fitoterápico PholiaNegra. Revista RX, 3(13).

Bianchi, M. D. L. P., \& Antunes, L. M. G. (1999). Radicais livres e os principais antioxidantes da dieta. Revista de nutrição, 12, 123-130. https://www.scielo.br/j/rn/a/bzHBTqBfJr8jmJn3ZXx9nMs/abstract/?format=html\&lang=pt\&stop=previous.

Brasil. (2006). Caderno de Atenção Básica-N14, Prevenção de Doenças Cardiovasculares, cerebrovasculares e renal crônica.

Brasil. (2002). Agência Nacional de Vigilância Sanitária. Resolução RDC no 2, de 07 de janeiro de 2002. Aprova o Regulamento Técnico de Substâncias Bioativas e probióticos isolados com alegação de Propriedade funcional e ou de Saúde. Brasília, DF. 
Caleiro, E. M. (2012). Efeito da suplementação do óleo de coco extra virgem sobre parâmetros clínicos e laboratoriais de ratos com obesidade induzida por dieta de cafeteria. http://bdtd.unoeste.br:8080/tede/handle/tede/669.

de Azevedo Campanella, L. C., da Silva, A. C., Freygang, J., \& Dal Magro, D. D. (2014). Efeito da suplementação de óleo de cártamo sobre o peso corporal, perfil lipídico, glicídico e antioxidante de ratos wistar induzidos a obesidade. Revista de Ciências Farmacêuticas Básica e Aplicada,35(1). http://rcfba.fcfar.unesp.br/index.php/ojs/article/view/167

Campos, S. D., Oliveira, M. B. P. P., \& ASAE. (2012). Suplementos alimentares para perda de peso: serão eficazes e seguros? Riscos $e$ Alimentos https://dspace.uevora.pt/rdpc/bitstream/10174/5222/1/Riscos\%20e\%20Alimentos\%20n\%C2\%BA\%203.pdf\#page=27.

de Carvalho, P. G., Machado, C. M. M., Moretti, C. L., \& Fonseca, M. E. D. N. (2006). Hortaliças como alimentos funcionais. Horticultura Brasileira, 24, 397-404. https://www.scielo.br/j/hb/a/L9XJQSJkrsncCgrKSGYQPHd/?format=pdf\&lang=pt.

Carvalho, J. (2004). Toxicidade pré-clínica: fitoterápicos e alimentos com propriedades funcionais ou de saúde Cozzolino, S. (2012). Nutracêuticos: o que significa. ABESO (Associação Brasileira para o estudo da Obesidade e da Síndrome Metabólica). Ed, 55, 5-7. https://www.researchgate.net/profile/SilviaCozzolino/publication/268385457_Nutraceuticos_o_que_Significa/links/56a771ce08ae860e02555f61/Nutraceuticos-o-que-Significa.pdf.

Ferreira, T. F. (2015). Revisão sistemática do óleo essencial da espécie Plectranthus amboinicus (Lour.) Spreng. https://repositorio.ufpb.br/jspui/handle/123456789/948.

de Araújo, M. B., \& de Mello, M. A. R. (2009). Exercício, estresse oxidativo e suplementação com creatina. RBNE-Revista Brasileira de Nutrição Esportiva, 3(15).

Duranti, M. (2006). Grain legume proteins and nutraceutical properties. Fitoterapia, 77(2), 67-82. https://www.sciencedirect.com/science/art icle/abs/pii/S0367326X0500239X.

Fernandes, H. P. (2014). Desenvolvimento do controle de qualidade de um produto nutracêutico na forma farmacêutica cápsula. http://repositorio.unesc.net/bitstream/1/2291/1/Henrique\%20Pacheco\%20Fernandes.pdf.

Ferrari, C. K. B., \& Torres, E. A. F. S. (2002). Novos compostos dietéticos com propriedades anticarcinogênicas. Revista Brasileira de Cancerologia, 48(3), 375-382.esearchgate.net/profile/Carlos-Ferrari/publication/353106532_Novos_compostos_dieteticos_com_propriedades_anticarcinogenicas/links/00463 538 4e16dd329a000000/Novos-compostos-dieteticos-com-propriedades-anticarcinogenicas.pdf.

Finner, A. M. (2012). Nutrition and hair: deficiencies and supplements. Dermatologic clinics, 31(1), 167-172. https://europepmc.org/article/med/23159185.

Gonçalves, G. M., \& Campos, P. M. (2013). Ácido ascórbico e ascorbil fosfato de magnésio na prevenção do envelhecimento cutâneo. Infarma-Ciências Farmacêuticas, 18(7/8), 3-6. http://revistas.cff.org.br/?journal=infarma\&page=article\&op=view\&path\%5B\%5D=242.

Hasenboehler, E., Williams, A., Leinhase, I., Morgan, S. J., Smith, W. R., Moore, E. E., \& Stahel, P. F. (2006). Metabolic changes after polytrauma: an imperative for early nutritional support. World journal of emergency surgery, 1(1), 1-7. https://wjes.biomedcentral.com/articles/10.1186/1749-7922-1-29.

Ictq. (2015). Instituto de ciência e tecnologia da qualidade. Nutracêuticos e suplementos são tendência nas farmácias. <http://ictq.com.br/portal/ .

Lima, M. S., Ribeiro, P. P., Medeiros, J., Silva, I. F., Medeiros, A. C., \& Dimenstein, R. (2012). Influence of postpartum supplementation with vitamin A on the levels of immunoglobulin A in human colostrum. Jornal de pediatria, 88, 115-118. https://www.scielo.br/j/jped/a/kMcBT nspBqPdesTGrh9m99J/abstract/?lang=en.

Lira, C. R. G., Zucco, F., Negrão, A. N., Silva, M. A. S., \& Murakami, F. S. (2009). Nutracêuticos: aspectos sobre segurança, controle de qualidade e legislação. Rev. Bras. Farm, 90(1), 45-49.

Lopes, M. C. D. C. P. (2013). Aconselhamento farmacêutico em suplementação alimentar (Doctoral dissertation, [sn]) https://bdigital.ufp.pt/handle/10284/4171.

Mannion, M. (1998). Nutraceutical revolution continues at foundation for innovation in medicine conference. Am J Nat Med, $5,30-3$.

Markle, B., May, E. J., \& Majumdar, A. P. (2010). Do nutraceutics play a role in the prevention and treatment of colorectal cancer? Cancer and metastasis reviews, 29(3), 395-404. https://link.springer.com/article/10.1007/s10555-010-9234-3.

Islas-Granillo, H., Borges-Yáñez, A., Fernández-Barrera, M. Á., Ávila-Burgos, L., Patiño-Marín, N., de Lourdes Márquez-Corona, M., \& Medina-Solís, C. E. (2017). Relationship of hyposalivation and xerostomia in Mexican elderly with socioeconomic, sociodemographic and dental factors. Scientific reports, 7(1), 1-8. https://www.nature.com/articles/srep40686.

Moraes, F. P. (2006). Alimentos funcionais e nutracêuticos: definições, legislação e benefícios à saúde. Revista eletrônica de farmácia, 3(2). https://www.revistas.ufg.br/ref/article/view/2082.

Nedel, D. R. (2005). Antioxidantes x radicais livres: a influência das vitaminas antioxidantes no retardo do envelhecimento cutâneo. 2005. 78f. MonografiaCurso de Graduação em Farmácia, Universidade Regional do Noroeste do Estado do Rio Grande do Sul, Ijuí.

Pineze, E. C., Consoni, R. D. C., \& Marques, L. C. (2003). Procedimentos operacionais de uma indústria farmacêutica: proposta de critérios de elaboração. Fármacos e Medicamentos, 23, 26-32.

Rajasekaran, A., Sivagnanam, G., \& Xavier, R. (2008). Nutraceuticals as therapeutic agents: A Review. Research Journal of Pharmacy and Technology, 1(4), 328-340. https://www.indianjournals.com/ijor.aspx?target=ijor:rjpt\&volume $=1 \&$ issue $=4 \&$ article $=006$

Rotta, M. A. (2003). Utilização do ácido ascórbico (vitamina C) pelos peixes. Embrapa Pantanal-Documentos (INFOTECA-E). https://www.infoteca.cnptia.embrapa.br/bitstream/doc/799021/1/DOC49.pdf. 
Sbaf. (2008). Sociedade Brasileira de Alimentos Funcionais. Alimentos que funcionam? Revista do Idec. <http://www.idec.org.br/uploads/revistas_

Schulze, B. N., Schultz, C., Ulbrich, A. Z., \& Bertin, R. L. (2014). Efeito da suplementação de óleo de cártamo sobre o perfil antropométrico e Lipídico de Mulheres com Excesso de Peso Praticantes de Exercício Físico. Revista Brasileira de Ciências da Saúde, 18(4), 89-96. esearchgate.net/profile/AndersonUlbrich/publication/287764203_Efeito_da_Suplementacao_de_Oleo_de_Cartamo_sobre_o_Perfil_Antropometrico_e_Lipidico_de_Mulheres_com_Excesso_d e_Peso_Praticantes_de_Exercicio_Fisico/links/57305c1e08ae744151910261/Efeito-da-Suplementacao-de-Oleo-de-Cartamo-sobre-o-Perfil-Antropometrico-eLipidico-de-Mulheres-com-Excesso-de-Peso-Praticantes-de-Exercicio-Fisico.pdf.

da Silva, M. E. M. (2013). Estudo de plantas medicinais utilizadas popularmente no tratamento da obesidade em Araranguá (Doctoral dissertation, Universidade Federal de Santa Catarina). https://uab.ufsc.br/biologia/files/2014/05/Maria-Eduarda-Moreira-da-Silva.pdf.

Sousa, C. M. D. M., Silva, H. R., Ayres, M. C. C., Costa, C. L. S. D., Araújo, D. S., Cavalcante, L. C. D., \& Chaves, M. H. (2007). Fenóis totais e atividade antioxidante de cinco plantas medicinais. Química nova, 30, 351-355. https://www.scielo.br/j/qn/a/4d6yvCyDmhHyfP9ZJqV6nDy/?format=html\&lang=pt.

Souza, M. A. F. D. (2008). Dos laboratórios aos pontos de venda: uma análise da trajetória dos alimentos funcionais e nutracêuticos e da sua repercussão sobre a questão agroalimentar. ttps://tede.ufrrj.br/handle/tede/699.

Stahl, W., \& Sies, H. (2003). Antioxidant activity of carotenoids. Molecular aspects of medicine, 24(6), 345-351. https://www.sciencedirect. com/science/article/abs/pii/S009829970300030X.

Stephen, I. D., Coetzee, V., Law Smith, M., \& Perrett, D. I. (2009). Skin blood perfusion and oxygenation colour affect perceived human health. PloS one, 4(4), e5083. https://journals.plos.org/plosone/article?id=10.1371/journal.pone.0005083.

Stringheta, P. C., Oliveira, T. T. D., Gomes, R. C., Amaral, M. D. P. H. D., Carvalho, A. F. D., \& Vilela, M. A. P. (2007). Políticas de saúde e alegações de propriedades funcionais e de saúde para alimentos no Brasil. Revista Brasileira de Ciências Farmacêuticas, 43, 181-194. https://www.scielo.br/j/rbcf/a/pk9QRgNjWqvW59jh3VFJChb/?lang=pt\&format=html.

Suleria, H. A. R., Osborne, S., Masci, P., \& Gobe, G. (2015). Marine-based nutraceuticals: An innovative trend in the food and supplement industries. Marine drugs, 13(10), 6336-6351. https://www.mdpi.com/1660-3397/13/10/6336.

da Silva Teixeira, G., Freire, R. A., da Fonseca, M. I. L., \& Bieski, I. G. C. (2014). Plantas medicinais, fitoterápicos e/ou nutracêuticos utilizados no controle da obesidade. FLOVET-Boletim do Grupo de Pesquisa da Flora, Vegetação e Etnobotânica, 1(6).

Valenzuela, A., Valenzuela, R., Sanhueza, J., \& Morales, G. (2014). Alimentos funcionales, nutraceúticos y foshu: ¿ vamos hacia un nuevo concepto de alimentación? Revista chilena de nutrición, 41(2), 198-204. https://scielo.conicyt.cl/scielo.php?pid=S0717-75182014000200011\&script=sci_arttext.

Wildman, R. E., Wildman, R., \& Wallace, T. C. (2016). Handbook of nutraceuticals and functional foods. CRC press. https://www.taylorfrancis.com/books/mono/10.1201/9781420006186/handbook-nutraceuticals-functional-foods-robert-wildman-robert-wildman-taylorwallace.

Wilkinson, J. A Indústria Alimentar e os Nutracêuticos. Workshop Identificação de Gargalos Tecnológicos na Agroindústria Paranaense: IPARDES, Curitiba, 2005. 129p.

Zeisel, S. H. (1999). Regulation of" nutraceuticals". https://www.science.org/doi/abs/10.1126/science.285.5435.1853. 